\title{
A GENERALIZATION OF LEVINGER'S THEOREM TO POSITIVE KERNEL OPERATORS
}

\author{
ROMAN DRNOVŠEK \\ Department of Mathematics, University of Ljubljana, Jadranska 19, SI-1000 Ljubljana, Slovenia \\ e-mail: roman.drnovsek@fmf.uni-lj.si
}

(Received 7 October, 2002; accepted 18 January, 2003)

\begin{abstract}
We prove some inequalities for the spectral radius of positive operators on Banach function spaces. In particular, we prove the following extension of Levinger's theorem. Let $K$ be a positive compact kernel operator on $L^{2}(X, \mu)$ with the spectral radius $r(K)$. Then the function $\phi$ defined by $\phi(t)=r\left(t K+(1-t) K^{*}\right)$ is non-decreasing on $\left[0, \frac{1}{2}\right]$. We also prove that $\left\|A+B^{*}\right\| \geq 2 \cdot \sqrt{r(A B)}$ for any positive operators $A$ and $B$ on $L^{2}(X, \mu)$.
\end{abstract}

2000 Mathematics Subject Classification. 47B34, 47B65, 47A10, 47A12, 47A63.

1. Introduction. In general there is no relation between the spectral radius of a sum of operators on a Banach space and the sum of the corresponding spectral radii, so that, under appropriate assumptions, any inequality between these two numbers might be interesting. In [5] we proved some inequalities for the spectral radius of a sum of positive compact kernel operators on a Banach function space. We thus extended the corresponding matrix results proved in [7]. In this article we show their further generalizations by removing several assumptions from the results in [5]. As an application of our main result we obtain an extension of Levinger's theorem to positive compact kernel operators on $L^{2}$-spaces. This beautiful result, stated without proof in [11], asserts that for a non-negative (square) matrix $A$ the function

$$
\phi(t)=r\left(t A+(1-t) A^{T}\right)
$$

is non-decreasing on $\left[0, \frac{1}{2}\right]$ and is non-increasing on $\left[\frac{1}{2}, 1\right]$. In particular, for all $t \in[0,1]$, the following inequality holds

$$
r\left(t A+(1-t) A^{T}\right) \geq r(A) .
$$

This theorem was generalized in Bapat [3], where an elementary proof is given. Recently, Alpin and Kolotilina [2, Theorem 7] further extended Bapat's result. Our Theorem 8 includes their extension as a special case. Finally, Theorem 10 proves an inequality that seems to be new even in the finite-dimensional case. For the theory of Banach function spaces and Banach lattices we refer the reader to the books [13], [12] and [1]. Here we shall recall some relevant facts.

Let $\mu$ be a $\sigma$-finite positive measure on a $\sigma$-algebra $\mathcal{M}$ of subsets of a non-void set $X$. Let $M(X, \mu)$ be the vector space of all equivalence classes of (almost everywhere equal) complex measurable functions on $X$. A Banach space $L \subseteq M(X, \mu)$ is called a Banach function space if $f \in L, g \in M(X, \mu)$, and $|g| \leq|f|$ imply that $g \in L$ and 
$\|g\| \leq\|f\|$. Throughout the paper, it is assumed that the dimension of $L$ is greater than one and that $X$ is the carrier of $L$, that is, there is no subset $Y$ of $X$ of strictly positive measure with the property that $f=0$ a.e. on $Y$ for all $f \in L$ (see [13]). The cone of positive elements in $L$ is denoted by $L_{+}$. A non-negative function $f \in L_{+}$is said to be strictly positive if $f(x)>0$ for almost all $x \in X$. The norm of $L$ is said to be a weakly Fatou norm if there exists a finite constant $k \geq 1$ such that $0 \leq f_{\tau} \uparrow f$ in $L$ implies that $\|f\| \leq k \cdot \sup _{\tau}\left\|f_{\tau}\right\|$.

By $L^{\prime}$ we denote the associate space (also called the Köthe dual) of all $g \in M(X, \mu)$ such that

$$
\varphi_{g}(f)=\int_{X} f g d \mu
$$

defines a bounded linear functional $\varphi_{g}$ on $L$. The space $L^{\prime}$ is also a Banach function space with respect to the associate norm $\|\cdot\|^{\prime}$ defined by

$$
\|g\|^{\prime}=\left\|\varphi_{g}\right\|=\sup \left\{\int_{X}|f g| d \mu: f \in L,\|f\| \leq 1\right\},
$$

and it may be considered as a closed subspace of the dual Banach lattice $L^{*}$. In view of the definition of $\|\cdot\|^{\prime}$ the following generalized Hölder's inequality holds

$$
\int_{X}|f g| d \mu \leq\|f\|\|g\|^{\prime}
$$

for $f \in L$ and $g \in L^{\prime}$. Note that the set $X$ is also the carrier of the associate space $L^{\prime}$, and $L^{\prime}$ separates points of $L$ (see [13, Theorem 112.1]). For any non-negative functions $f$ and $g$ on $X$ we introduce the following notation

$$
\langle f, g\rangle=\int_{X} f g d \mu .
$$

For brevity, the integration over the whole set $X$ will be denoted by $\int d \mu(x)$ or even $\int d x$.

By an operator on a Banach function space $L$ we always mean a linear operator on $L$. The spectrum and the spectral radius of a bounded operator $T$ on $L$ are denoted by $\sigma(T)$ and $r(T)$, respectively. An operator $T$ on $L$ is said to be positive if $T f \in L_{+}$ for all $f \in L_{+}$. Given operators $S$ and $T$ on $L$, we write $S \geq T$ if the operator $S-T$ is positive. It should be recalled that a positive operator $T$ on $L$ is automatically bounded and that $r(T)$ belongs to the spectrum of $T$. An operator $K$ on $L$ is called a kernel operator if there exists a $\mu \times \mu$-measurable function $k(x, y)$ on $X \times X$ such that, for all $f \in L$ and for almost all $x \in X$,

$$
\int_{X}|k(x, y) f(y)| d \mu(y)<\infty \text { and }(K f)(x)=\int_{X} k(x, y) f(y) d \mu(y) .
$$

One can check that a kernel operator $K$ is positive iff its kernel $k$ is non-negative almost everywhere. We say that $K$ is reducible if there exists a set $A \in \mathcal{M}$ such that $\mu(A)>0$, $\mu\left(A^{c}\right)>0$ and $k=0$ a.e. on $A \times A^{c}$. Otherwise, if there is no such set, $K$ is said to be irreducible.

Let $K$ be a positive kernel operator on $L$ with kernel $k$. It is easily seen that $L^{\prime}$ is invariant under the adjoint operator $K^{*}$. We denote by $K^{\prime}$ the restriction of $K^{*}$ to $L^{\prime}$. 
One can show [13, Section 97] that $K^{\prime}$ is also a positive kernel operator with the kernel $k^{\prime}(x, y)=k(y, x)(x, y \in X)$. The following important observation was already stated in [6] for general Banach lattices.

Proposition 1. Let $L$ be a Banach function space with a weakly Fatou norm. If $K$ is a kernel operator on $L$, then $r\left(K^{\prime}\right)=r(K)$.

Proof. It follows from [13, Theorem 107.7] (see also the equality (2) on p. 393 of [13]) that the space $L$ can be (not necessarily isometrically) embedded into $\left(L^{\prime}\right)^{\prime}$ as a Banach space. Then we have $r(K) \geq r\left(K^{\prime}\right) \geq r\left(\left(K^{\prime}\right)^{\prime}\right) \geq r(K)$, and so $r\left(K^{\prime}\right)=r(K)$.

The following important result is contained in [9, Theorems 4.13 and 3.14].

THEOREM 2. Let $K$ be an irreducible positive kernel operator on a Banach function space $L$ such that $r(K)$ is a pole of the resolvent $(\lambda-K)^{-1}$. Then $r(K)>0, r(K)$ is an eigenvalue of $K$ of algebraic multiplicity one, and the corresponding eigenspace is spanned by a strictly positive function.

It is well known that the assumption that $r(K)$ is a pole of the resolvent $(\lambda-K)^{-1}$ is satisfied if some power of $K$ is a compact operator. In this case Theorem 2 is known as the theorem of Jentzsch and Perron (see [9, Theorem 5.2]).

We will also need the following simple result.

Proposition 3. Assume that a positive operator $T$ on a Banach function space $L$ is the norm limit of a sequence $\left\{T_{n}\right\}_{n \in \mathbb{N}}$ of positive operators on $L$ such that $T_{1} \geq T_{2} \geq \ldots \geq T$. Then

$$
r(T)=\lim _{n \rightarrow \infty} r\left(T_{n}\right) .
$$

Proof. The sequence $\left\{r\left(T_{n}\right)\right\}_{n \in \mathbb{N}}$ is non-increasing and bounded below by $r(T)$, so that $r(T) \leq \lim _{n \rightarrow \infty} r\left(T_{n}\right)$. Since the spectral radius is upper semicontinuous, the equality holds in this inequality.

2. General Banach function spaces. Throughout this section, let $L$ be a Banach function space with a weakly Fatou norm. For brevity, we denote by $L_{++}^{\infty}(X, \mu)$ the set of all strictly positive functions $f \in L^{\infty}(X, \mu)_{+}$satisfying $1 / f \in L^{\infty}(X, \mu)_{+}$. For $d \in L^{\infty}(X, \mu)_{+}$the multiplication operator $D$ is a positive operator on $L$ defined by $D f=d f$. Clearly, $D$ is invertible iff $d \in L_{++}^{\infty}(X, \mu)$.

The following lemma that extends [5, Lemma 2.2] is needed in the proof of Theorem 5.

LEMMA 4. Let $K$ be a positive kernel operator on $L$ with $r(K)=1$. Let $d$ and $e$ be strictly positive functions in $L_{++}^{\infty}(X, \mu)$, and let $D$ and $E$ be the corresponding multiplication operators on $L$. Let $f \in L_{+}$and $g \in L_{+}^{\prime}$ be strictly positive functions such that $K f$ is a strictly positive function satisfying

$$
\frac{K f}{f}=\frac{K^{\prime} g}{g} \text { and }\langle K f, g\rangle=1 .
$$

Then

$$
\langle D K E u, v\rangle \geq \exp \left(\int_{X} K f g \log (d e) d \mu\right)
$$


for any $u \in L_{+}$and for any nonnegative measurable function $v$ on $X$ satisfying $u v=f g$. If, in addition, $\langle K u, v\rangle<\infty$, then

$$
\langle K u, v\rangle \geq \exp \left(\int_{X} K f g \log \left(\frac{K u}{u} \frac{f}{K f}\right) d \mu\right) \geq 1 .
$$

Proof. Since $\langle K f, g\rangle=1$, the integral in (1) exists, while it will be seen below that the integral in (2) exists provided $\langle K u, v\rangle<\infty$. In fact, there is no loss of generality in assuming that $\langle D K E u, v\rangle<\infty$, and consequently, $\langle K u, v\rangle<\infty$, since it holds

$$
\langle K u, v\rangle \leq\|1 / d\|_{\infty} \cdot\|1 / e\|_{\infty} \cdot\langle D K E u, v\rangle .
$$

We will first show the right-hand inequality in (2), that is

$$
\int_{X} K f g \log \left(\frac{K u}{u} \frac{f}{K f}\right) d \mu \geq 0 .
$$

We consider the special case when $v \in L_{+}^{\prime}$. For almost all $x \in X$ we define the probability measure on $\mathcal{M}$ by

$$
v_{x}(A)=\frac{1}{(K f)(x)} \int_{A} k(x, y) f(y) d y,
$$

where $k$ is the kernel of $K$. Using the estimate $|\log (t)| \leq t+\frac{1}{t}(t>0)$ we obtain that

$$
\int K f g\left|\log \left(\frac{u}{f}\right)\right| d \mu \leq \int K f g\left(\frac{u}{f}+\frac{f}{u}\right) d \mu=\left\langle u, K^{\prime} g\right\rangle+\langle K f, v\rangle<\infty .
$$

Now, we have

$$
\begin{aligned}
\int(K f)(y) g(y) \log \left(\frac{u(y)}{f(y)}\right) d y & =\int f(y)\left(K^{\prime} g\right)(y) \log \left(\frac{u(y)}{f(y)}\right) d y \\
& =\int f(y) \log \left(\frac{u(y)}{f(y)}\right)\left(\int k(x, y) g(x) d x\right) d y .
\end{aligned}
$$

Because of (4) we can use Fubini's theorem to get

$$
\begin{aligned}
\int(K f)(y) g(y) \log \left(\frac{u(y)}{f(y)}\right) d y & =\int g(x)\left(\int k(x, y) f(y) \log \left(\frac{u(y)}{f(y)}\right) d y\right) d x \\
& =\int(K f)(x) g(x)\left(\int \log \left(\frac{u(y)}{f(y)}\right) d v_{x}(y)\right) d x .
\end{aligned}
$$

Then, an application of Jensen's inequality gives the inequality

$$
\begin{aligned}
\int(K f)(y) g(y) \log \left(\frac{u(y)}{f(y)}\right) d y & \leq \int(K f)(x) g(x) \log \left(\int \frac{u(y)}{f(y)} d v_{x}(y)\right) d x \\
& =\int(K f)(x) g(x) \log \left(\frac{(K u)(x)}{(K f)(x)}\right) d x,
\end{aligned}
$$

from which (3) follows. To prove the general case, define sequences $\left\{u_{n}\right\}_{n \in \mathbb{N}}$ and $\left\{v_{n}\right\}_{n \in \mathbb{N}}$ of strictly positive functions by $u_{n}=u+f / n$ and $v_{n}=f g / u_{n}$. Since $v_{n} \leq n g$, we have 
$v_{n} \in L_{+}^{\prime}$, and so

$$
\int_{X} K f g \log \left(\frac{K u_{n}}{u_{n}} \frac{f}{K f}\right) d \mu \geq 0
$$

by the special case of (3). Since

$$
\frac{K u_{n}}{u_{n}}-\frac{K f}{f}=\frac{u}{u_{n}}\left(\frac{K u}{u}-\frac{K f}{f}\right)
$$

it holds that

$$
\left\{x \in X: \frac{\left(K u_{n}\right)(x)}{u_{n}(x)} \geq \frac{(K f)(x)}{f(x)}\right\}=\left\{x \in X: \frac{(K u)(x)}{u(x)} \geq \frac{(K f)(x)}{f(x)}\right\},
$$

and the sequence $\left\{\frac{K u_{n}}{u_{n}}\right\}_{n \in \mathbb{N}}$ is non-decreasing on this set. Then, by the Monotone Convergence Theorem,

$$
\lim _{n \rightarrow \infty} \int_{X} K f g \log ^{+}\left(\frac{K u_{n}}{u_{n}} \frac{f}{K f}\right) d \mu=\int_{X} K f g \log ^{+}\left(\frac{K u}{u} \frac{f}{K f}\right) d \mu,
$$

where the limit is finite. Namely, using the inequality $\log ^{+} t \leq t(t>0)$ we obtain that

$$
0 \leq \int_{X} K f g \log ^{+}\left(\frac{K u}{u} \frac{f}{K f}\right) d \mu \leq \int_{X} K f g \frac{K u}{u} \frac{f}{K f} d \mu=\langle K u, v\rangle<\infty
$$

This shows that the integral in (3) is defined (and its value belongs to $[-\infty, \infty)$ ). Similarly, we obtain that

$$
\lim _{n \rightarrow \infty} \int_{X} K f g \log ^{-}\left(\frac{K u_{n}}{u_{n}} \frac{f}{K f}\right) d \mu=\int_{X} K f g \log ^{-}\left(\frac{K u}{u} \frac{f}{K f}\right) d \mu,
$$

which together with (6) gives that

$$
\lim _{n \rightarrow \infty} \int_{X} K f g \log \left(\frac{K u_{n}}{u_{n}} \frac{f}{K f}\right) d \mu=\int_{X} K f g \log \left(\frac{K u}{u} \frac{f}{K f}\right) d \mu .
$$

In view of (5) this completes the proof of (3).

We now define the probability measure $\lambda$ on $\mathcal{M}$ by

$$
\lambda(A)=\int_{A} K f g d \mu .
$$

An application of Jensen's inequality gives that

$$
\begin{aligned}
\log (\langle K u, v\rangle) & =\log \left(\int \frac{K u}{u} \frac{f}{K f} d \lambda\right) \geq \int \log \left(\frac{K u}{u} \frac{f}{K f}\right) d \lambda \\
& =\int K f g \log \left(\frac{K u}{u} \frac{f}{K f}\right) d \mu,
\end{aligned}
$$


so that the left-hand inequality holds in (2). Similarly, we have

$$
\begin{aligned}
\log (\langle D K E u, v\rangle) & =\log \left(\int d e \frac{K(E u)}{E u} \frac{f}{K f} d \lambda\right) \geq \int \log \left(d e \frac{K(E u)}{E u} \frac{f}{K f}\right) d \lambda \\
& =\int K f g \log (d e) d \mu+\int K f g \log \left(\frac{K(E u)}{E u} \frac{f}{K f}\right) d \mu .
\end{aligned}
$$

Since the last integral is non-negative by (3), this gives (1).

The following result extends Theorems 2.4 and 2.6 in [5]. Its finite-dimensional version was shown in [7, Theorem 2.3].

THEOREM 5. Let $K_{1}, K_{2}, \ldots, K_{n}$ be positive kernel operators on L. Assume that $f_{1}$, $f_{2}, \ldots, f_{n} \in L_{+}$and $g_{1}, g_{2}, \ldots, g_{n} \in L_{+}^{\prime}$ are strictly positive functions satisfying

$$
K_{i} f_{i}=r\left(K_{i}\right) f_{i}, \quad K_{i}^{\prime} g_{i}=r\left(K_{i}\right) g_{i}
$$

and be normalized so that

$$
f_{i} \cdot g_{i}=h(i=1,2, \ldots, n) \text { and } \int_{X} h d \mu=1 .
$$

Furthermore, let $d_{1}, \ldots, d_{n}$ and $e_{1}, \ldots, e_{n}$ be in $L^{\infty}(X, \mu)_{+}$, and let $D_{1}, \ldots, D_{n}$ and $E_{1}, \ldots, E_{n}$ be the corresponding multiplication operators on $L$. Then

$$
r\left(\sum_{i=1}^{n} D_{i} K_{i} E_{i}\right) \geq \sum_{i=1}^{n} r\left(K_{i}\right) \exp \left(\int_{X} h \log \left(d_{i} e_{i}\right) d \mu\right)
$$

adopting the convention $\exp (-\infty)=0$. In particular, for all positive numbers $t_{1}, \ldots, t_{n}$,

$$
r\left(t_{1} K_{1}+\ldots+t_{n} K_{n}\right) \geq t_{1} r\left(K_{1}\right)+\ldots+t_{n} r\left(K_{n}\right) .
$$

Proof. If, for some $i, d_{i} e_{i}=0$ on the set of positive measure, then $\int_{X} h \log \left(d_{i} e_{i}\right)$ $d \mu=-\infty$, which together with the monotonicity of the spectral radius convinces us that there is no loss of generality in assuming that $\left\{d_{i}\right\}_{i=1}^{n}$ and $\left\{e_{i}\right\}_{i=1}^{n}$ are strictly positive functions. Also, we may assume that $r\left(K_{i}\right)>0$ for all $i$.

Consider first the case when $\left\{d_{i}\right\}_{i=1}^{n}$ and $\left\{e_{i}\right\}_{i=1}^{n}$ are in $L_{++}^{\infty}(X, \mu)$. Denote $K=$ $D_{1} K_{1} E_{1}+\ldots+D_{n} K_{n} E_{n}$, pick $\lambda>r(K)$, and set

$$
u=(\lambda-K)^{-1} f_{1}=\sum_{j=0}^{\infty} \lambda^{-j-1} K^{j} f_{1} .
$$

Then $u$ is a strictly positive function in $L$ satisfying $K u \leq \lambda u$. Denoting $v=h / u$ we apply (1) of Lemma 4 for the operator $K_{i} / r\left(K_{i}\right), i=1, \ldots, n$, to get

$$
\left\langle D_{i} K_{i} E_{i} u, v\right\rangle \geq r\left(K_{i}\right) \exp \left(\int_{X} h \log \left(d_{i} e_{i}\right) d \mu\right) .
$$

Summing over $i$ gives the inequality

$$
\sum_{i=1}^{n} r\left(K_{i}\right) \exp \left(\int_{X} h \log \left(d_{i} e_{i}\right) d \mu\right) \leq \sum_{i=1}^{n}\left\langle D_{i} K_{i} E_{i} u, v\right\rangle=\langle K u, v\rangle \leq\langle\lambda u, v\rangle=\lambda .
$$

Since this is true for any $\lambda>r(K)$, the inequality (7) follows. 
To remove the assumptions on $\left\{d_{i}\right\}_{i=1}^{n}$ and $\left\{e_{i}\right\}_{i=1}^{n}$, define $d_{i}^{(m)}=\max \left\{d_{i}, \frac{1}{m}\right\}$ and $e_{i}^{(m)}=\max \left\{e_{i}, \frac{1}{m}\right\}(m \in \mathbb{N}, i=1, \ldots, n)$, and let $D_{i}^{(m)}$ and $E_{i}^{(m)}$ be the corresponding multiplication operators on $L$. Then, by the above,

$$
r\left(\sum_{i=1}^{n} D_{i}^{(m)} K_{i} E_{i}^{(m)}\right) \geq \sum_{i=1}^{n} r\left(K_{i}\right) \exp \left(\int_{X} h \log \left(d_{i}^{(m)} e_{i}^{(m)}\right) d \mu\right) .
$$

When $m$ tends to infinity, the left-hand side approaches $r(K)$ by Proposition 3, while

$$
\lim _{m \rightarrow \infty} \int_{X} h \log \left(d_{i}^{(m)} e_{i}^{(m)}\right) d \mu=\int_{X} h \log \left(d_{i} e_{i}\right) d \mu
$$

by the Monotone Convergence Theorem (for decreasing sequences). This yields the inequality (7), and the proof is finished.

A glance at the proof above shows that Theorem 5 also holds in the case when some operators of $K_{1}, K_{2}, \ldots, K_{n}$ are positive multiples of the identity operator, or in other words, every $K_{i}$ is a sum of a positive kernel operator and a non-negative multiple of the identity.

Given a positive operator $T$ on $L$, let $\mathcal{P}_{+}(T)$ denote the set of all functions $p(z)=$ $\sum_{k=0}^{\infty} a_{k} z^{k}$ such that $a_{k} \geq 0$ for all $k$ and the convergence radius of $p$ is greater than $r(T)$. Using the spectral mapping theorem one can show easily that $r(p(T))=p(r(T))$ for all $p \in \mathcal{P}_{+}(T)$.

THEOREM 6. Under the assumptions of Theorem 5 , let $p_{i} \in \mathcal{P}_{+}\left(K_{i}\right)$ for $i=1, \ldots, n$. Then

$$
r\left(p_{1}\left(K_{1}\right)+\ldots+p_{n}\left(K_{n}\right)\right) \geq p_{1}\left(r\left(K_{1}\right)\right)+\ldots+p_{n}\left(r\left(K_{n}\right)\right) .
$$

In particular, if $s_{i}>r\left(K_{i}\right)$ for $i=1, \ldots, n$, then

$$
r\left(\left(s_{1}-K_{1}\right)^{-1}+\ldots+\left(s_{n}-K_{n}\right)^{-1}\right) \geq \frac{1}{s_{1}-r\left(K_{1}\right)}+\ldots+\frac{1}{s_{n}-r\left(K_{n}\right)} .
$$

Proof. We first claim that every $p_{i}\left(K_{i}\right), i=1, \ldots, n$, is the sum of a kernel operator and a non-negative multiple of the identity operator $I$. If $p_{i}(z)=\sum_{k=0}^{\infty} a_{k} z^{k}$ with $a_{k} \geq 0$, then $p_{i}\left(K_{i}\right)-a_{0} I$ is the limit (in norm and in order) of an increasing sequence of kernel operators. It follows that it is a kernel operator (see e.g. [13, Theorem 94.5]). This proves our claim. Now, according to the remark following the proof of Theorem 5 we may apply the inequality (8) of Theorem 5 for operators $p_{1}\left(K_{1}\right), \ldots, p_{n}\left(K_{n}\right)$ to get $r\left(p_{1}\left(K_{1}\right)+\ldots+p_{n}\left(K_{n}\right)\right) \geq r\left(p_{1}\left(K_{1}\right)\right)+\ldots+r\left(p_{n}\left(K_{n}\right)\right)=p_{1}\left(r\left(K_{1}\right)\right)+\ldots+p_{n}\left(r\left(K_{n}\right)\right)$.

As an extension of Theorem 4.2 in [8] we now show that the inequality (7) of Theorem 5 for $n=1$ can be improved if the operator is of the form $(s-K)^{-1}$, where $s>r(K)$.

THEOREM 7. Let $K$ be a positive operator on $L$ with $r(K)>0$ that is a sum of a positive kernel operator and a non-negative multiple of the identity. Assume that $f \in L_{+}$and $g \in L_{+}^{\prime}$ are strictly positive functions satisfying $K f=r(K) f, K^{\prime} g=r(K) g$ and $\langle f, g\rangle=1$. 
Let $d$ be in $L^{\infty}(X, \mu)_{+}$, and let $D$ be the corresponding multiplication operator on $L$. Then

$$
r(D K) \geq r(K) \exp \left(\int_{X} f g \log (d) d \mu\right) .
$$

Furthermore, for $s>r(K)$ it holds

$$
r\left(D(s-K)^{-1}\right) \geq r\left((s-K)^{-1}\right)\left(\int_{X} f g d d \mu\right) .
$$

Proof. The inequality (9) is a special case of (7). Denote $T=(s-K)^{-1}$ and pick $\lambda>r(D T)$. Then $w=(\lambda-D T)^{-1} f$ is a strictly positive function in $L$ satisfying $D T w \leq$ $\lambda w$. Set $u=T w$ and $v=f \cdot g / u$. If we apply (2) of Lemma 4 for the operator $K / r(K)$, we obtain that $\langle K u, v\rangle \geq r(K)$, and so

$$
\left\langle T^{-1} u, v\right\rangle=\langle(s-K) u, v\rangle \leq s-r(K)=\frac{1}{r(T)} .
$$

On the other hand, since $\lambda T^{-1} u=\lambda w \geq D T w=d u$, we have $\lambda\left\langle T^{-1} u, v\right\rangle \geq\langle d u, v\rangle$. It follows that $\lambda \geq r(T)\langle d u, v\rangle$ which implies (10).

Observe that (10) is really a sharpening of (9) for the special class of positive operators, since

$$
\exp \left(\int_{X} f g \log (d) d \mu\right) \leq \int_{X} f g d d \mu
$$

by Jensen's inequality. Also, simple examples show that in (9) $\exp \left(\int_{X} f g \log (d) d \mu\right)$ can not be replaced by $\int_{X} f g d d \mu$. (Consider $K=\left[\begin{array}{ll}0 & 1 \\ 1 & 0\end{array}\right]$ on $L=\mathbb{C}^{2}$.)

3. $L^{2}$-spaces. In [5] we proved an extension of Levinger's inequality to positive kernel operators on $L^{2}$-spaces. Unfortunately, we were able to show it only under some assumptions on the kernel of the operator. We now show that these assumptions are redundant, as we expected. In the finite-dimensional case this result was proved in [2, Theorem 7].

THEOREM 8. Let $K$ be a positive kernel operator on $L^{2}(X, \mu)$ such that $r(K)$ is an isolated point of $\sigma(K)$ and the corresponding Riesz idempotent has finite rank. Let $d \in$ $L_{++}^{\infty}(X, \mu)$ be a strictly positive function, and let $D$ be the corresponding multiplication operator on $L^{2}(X, \mu)$. Then, for any $t \in[0,1]$,

$$
r\left(t D K D^{-1}+(1-t) K^{*}\right) \geq r(K) .
$$

If, in addition, the operator $K$ is compact and if $\phi:[0,1] \rightarrow[0, \infty)$ is defined by

$$
\phi(t)=r\left(t D K D^{-1}+(1-t) K^{*}\right),
$$

then $\phi$ is non-decreasing on $\left[0, \frac{1}{2}\right]$ and is non-increasing on $\left[\frac{1}{2}, 1\right]$.

Proof. Consider first the case when $D=I$, the identity on $L$. If $K$ is irreducible, then by Theorem 2 there exist strictly positive functions $f, g \in L^{2}(X, \mu)$ satisfying 
$K f=r(K) f, K^{*} g=r(K) g$ and $\langle f, g\rangle=1$, and the inequality (11) follows from Theorem 5 with $K_{1}=K, K_{2}=K^{*}, f_{1}=g_{2}=f$ and $g_{1}=f_{2}=g$. For general $K$ pick any strictly positive function $u \in L^{2}(X, \mu)$. (Such functions exist because the measure $\mu$ is $\sigma$-finite.) Denote by $K_{0}$ an irreducible kernel operator with strictly positive kernel $u(x) u(y)(x, y \in X)$. For each $m \in \mathbb{N}$ define an irreducible positive kernel operator on $L^{2}(X, \mu)$ by $K_{m}=K+\frac{1}{m} K_{0}$. Then $r\left(K_{m}\right) \geq r(K)$, and the left (and, similarly, the right) essential spectra of $K_{m}$ and $K$ coincide. Now, Proposition XI.6.9 and Theorem XI.6.8 of [4] imply that $r\left(K_{m}\right)$ is an isolated point of $\sigma\left(K_{m}\right)$ and the corresponding Riesz idempotent has finite rank. By the first part of the proof, we then have

$$
r\left(t K+(1-t) K^{*}+\frac{1}{m} K_{0}\right)=r\left(t\left(K+\frac{1}{m} K_{0}\right)+(1-t)\left(K+\frac{1}{m} K_{0}\right)^{*}\right) \geq r\left(K+\frac{1}{m} K_{0}\right) .
$$

Letting $m \rightarrow \infty$ we get $\phi(t) \geq r(K)$ by Proposition 3, which proves (11) in the case $D=I$. Since $\phi(t)=\phi(1-t)$, it remains to show in this special case that $\phi$ is nondecreasing on $\left[0, \frac{1}{2}\right]$ provided $K$ is compact. Let $0 \leq t<s \leq \frac{1}{2}$. Then, by (11),

$$
\begin{aligned}
\phi(t) & \leq r\left(u\left(t K+(1-t) K^{*}\right)+(1-u)\left(t K+(1-t) K^{*}\right)^{*}\right) \\
& =r\left((2 u t-u-t+1) K+(t+u-2 u t) K^{*}\right)
\end{aligned}
$$

for all $u \in[0,1]$. Put $u=\frac{1-s-t}{1-2 t}$ to obtain that $\phi(t) \leq r\left(s K+(1-s) K^{*}\right)=\phi(s)$.

The general case follows from the special one. To show this, let $E$ be the multiplication operator on $L$ the multiplier of which is $\sqrt{d}$, so that $E^{2}=D$. Introducing the notation

$$
\phi_{K, D}(t)=r\left(t D K D^{-1}+(1-t) K^{*}\right)
$$

we have, for all $t \in[0,1]$,

$$
\begin{aligned}
\phi_{K, D}(t) & =r\left(E\left(t E K E^{-1}+(1-t) E^{-1} K^{*} E\right) E^{-1}\right) \\
& =r\left(t E K E^{-1}+(1-t)\left(E K E^{-1}\right)^{*}\right)=\phi_{E K E^{-1}, I}(t) .
\end{aligned}
$$

Since $\phi_{E K E^{-1}, I}(t) \geq r\left(E K E^{-1}\right)=r(K)$ by the special case, (11) follows. If, in addition, $K$ is compact, then $\phi_{E K E^{-1}, I}$ is non-decreasing on $\left[0, \frac{1}{2}\right]$ and is non-increasing on $\left[\frac{1}{2}, 1\right]$ by the special case, and so the same is also true for $\phi_{K, D}$. This completes the proof.

We do not know whether Theorem 8 is valid for every positive operator $K$ on $L^{2}(X, \mu)$. However, we shall show below that for $t=1 / 2$ the inequality (11) holds for all positive operators on $L^{2}(X, \mu)$. To do this, we recall that the numerical radius $w(A)$ of a bounded operator $A$ on $L^{2}(X, \mu)$ is defined by

$$
w(A)=\sup \left\{|\langle A f, f\rangle|: f \in L^{2}(X, \mu),\|f\|_{2}=1\right\} .
$$

If, in addition, $A$ is positive, then we have

$$
w(A)=\sup \left\{\langle A f, f\rangle: f \in L^{2}(X, \mu)_{+},\|f\|_{2}=1\right\} .
$$

Indeed, this follows from the estimate

$$
|\langle A f, f\rangle| \leq \int_{X}|A f||f| d \mu \leq\langle A|f|,|f|\rangle
$$


that holds for any $f \in L^{2}(X, \mu)$. It is well known [10] that

$$
r(A) \leq w(A) \leq\|A\|
$$

for all bounded operators $A$ on $L^{2}(X, \mu)$.

THEOREM 9. Let $A$ be a positive operator on $L^{2}(X, \mu)$. Then, for any $t \in[0,1]$,

$$
\|A\| \geq\left\|t A+(1-t) A^{*}\right\| \geq w\left(t A+(1-t) A^{*}\right)=w(A) \geq r(A)
$$

and

$$
\left\|\left(t A+(1-t) A^{*}\right)^{2}\right\| \geq w\left(\left(t A+(1-t) A^{*}\right)^{2}\right) \geq w\left(A^{2}\right) \geq(r(A))^{2} .
$$

Furthermore, if $d$ is in $L_{++}^{\infty}(X, \mu)$ and $D$ is the corresponding multiplication operator on $L^{2}(X, \mu)$, then

$$
r\left(D A D^{-1}+A^{*}\right) \geq 2 r(A)
$$

Proof. The equality in (12) follows from

$$
\left\langle\left(t A+(1-t) A^{*}\right) f, f\right\rangle=t\langle A f, f\rangle+(1-t)\langle f, A f\rangle=\langle A f, f\rangle,
$$

which holds for all $f \in L^{2}(X, \mu)_{+}$. The remaining inequalities in (12) are clear. Similarly, only the second inequality in (13) needs a proof. This relation is a consequence of the following inequality

$$
\left\langle\left(t A+(1-t) A^{*}\right)^{2} f, f\right\rangle \geq\left\langle A^{2} f, f\right\rangle
$$

that holds for every $f \in L^{2}(X, \mu)_{+}$, since it is equivalent to $t(1-t)\left\|A f-A^{*} f\right\|_{2}^{2} \geq 0$. Setting $t=1 / 2$ in (12) we obtain (14) in the case $D=I$, since $r\left(A+A^{*}\right)=w\left(A+A^{*}\right)=$ $\left\|A+A^{*}\right\|$. The general case can be obtained from the special one as in the proof of Theorem 8. Namely, if $E$ is the multiplication operator on $L$ with the multiplier $\sqrt{d}$, then

$$
\begin{aligned}
r\left(D A D^{-1}+A^{*}\right) & =r\left(E\left(E A E^{-1}+E^{-1} A^{*} E\right) E^{-1}\right) \\
& =r\left(E A E^{-1}+\left(E A E^{-1}\right)^{*}\right) \geq 2 r\left(E A E^{-1}\right)=2 r(A) .
\end{aligned}
$$

An application of Berberian's trick concerning $2 \times 2$ operator matrices gives the following result which seems to be new even in the finite-dimensional case.

THEOREM 10. Let $A$ and $B$ be positive operators on $L^{2}(X, \mu)$. Then

$$
\left\|A+B^{*}\right\| \geq 2 \cdot \sqrt{r(A B)} .
$$

If, in addition, $A$ and $B$ are compact kernel operators, then, for each $t \in[0,1]$,

$$
\max \left\{\left\|t A+(1-t) B^{*}\right\|,\left\|t B+(1-t) A^{*}\right\|\right\} \geq \sqrt{r(A B)} .
$$

Proof. Let $T$ be a positive operator on $L^{2}(X, \mu) \oplus L^{2}(X, \mu)$ defined by $2 \times 2$ operator matrix

$$
T=\left[\begin{array}{cc}
0 & A \\
B & 0
\end{array}\right] .
$$


Then $r\left(T+T^{*}\right)=\left\|T+T^{*}\right\|=\left\|A+B^{*}\right\|$ and $(r(T))^{2}=r\left(T^{2}\right)=r(A B)$. By (14), we obtain that

$$
\left\|A+B^{*}\right\|=r\left(T+T^{*}\right) \geq 2 r(T)=2 \sqrt{r(A B)} .
$$

If, in addition, $A$ and $B$ are compact kernel operators, then $T$ is a compact kernel operator as well. Then, for each $t \in[0,1]$,

$$
\begin{aligned}
\sqrt{r(A B)} & =r(T) \leq r\left(t T+(1-t) T^{*}\right) \leq\left\|t T+(1-t) T^{*}\right\| \\
& =\max \left\{\left\|t A+(1-t) B^{*}\right\|,\left\|t B+(1-t) A^{*}\right\|\right\},
\end{aligned}
$$

where we have used (11). This completes the proof.

AcKnowledgment. This work was supported in part by the Ministry of Education, Science and Sport of Slovenia.

\section{REFERENCES}

1. C. D. Aliprantis and O. Burkinshaw, Positive operators (Academic Press, Orlando, 1985).

2. Yu. A. Alpin and L. Yu. Kolotilina, Inequalities for the Perron root related to Levinger's theorem, Lin. Alg. Appl. 283 (1998), 99-113. 248.

3. R. B. Bapat, Two inequalities for the Perron root, Lin. Alg. Appl. 85 (1987), 241-

4. J. B. Conway, A course in functional analysis (Springer-Verlag, 1990).

5. R. Drnovšek, Spectral inequalities for compact integral operators on Banach function spaces, Math. Proc. Camb. Phil. Soc. 112 (1992), 589-598.

6. R. Drnovšek, Bounds for the spectral radius of positive operators, Comment. Math. Univ. Carolinae 41 (2000), 459-467.

7. L. Elsner and C. R. Johnson, Nonnegative Matrices, Zero Patterns, and Spectral Inequalities, Lin. Alg. Appl. 120 (1989), 225-236.

8. S. Friedland and S. Karlin, Some inequalities for the spectral radius of non-negative matrices and applications, Duke Math. J. 42 (1975), 459-490.

9. J. J. Grobler, Spectral theory on Banach lattices, in Operator theory in function spaces and Banach lattices, Oper. Theory Adv. Appl. No. 75 (Birkhäuser, 1995).

10. K. E. Gustafson and D. K. M. Rao, Numerical range. The field of values of linear operators and matrices, Universitext (Springer-Verlag, 1997).

11. B. W. Levinger, An inequality for nonnegative matrices, Notices Amer. Math. Soc. 17 (1970), 260.

12. P. Meyer-Nieberg, Banach lattices (Springer-Verlag, 1991).

13. A. C. Zaanen, Riesz spaces II (North Holland, 1983). 